\title{
Efficient Dual Algorithms for Image Segmentation Using TV-Allen-Cahn Type Models
}

\author{
Li-Lian Wang* and Ying Gu \\ Division of Mathematical Sciences, School of Physical and Mathematical Sciences, \\ Nanyang Technological University, 637371, Singapore.
}

Received 22 November 2009; Accepted (in revised version) 29 July 2010

Available online 13 October 2010

\begin{abstract}
Variational image segmentation based on the Mumford and Shah model [31], together with implementation by the piecewise constant level-set method (PCLSM) [26], leads to fully nonlinear Total Variation (TV)-Allen-Cahn equations. The commonlyused numerical approaches usually suffer from the difficulties not only with the nondifferentiability of the TV-term, but also with directly evolving the discontinuous piecewise constant-structured solutions. In this paper, we propose efficient dual algorithms to overcome these drawbacks. The use of a splitting-penalty method results in TVAllen-Cahn type models associated with different "double-well" potentials, which allow for the implementation of the dual algorithm of Chambolle [8]. Moreover, we present a new dual algorithm based on an edge-featured penalty of the dual variable, which only requires to solve a vectorial Allen-Cahn type equation with linear $\nabla$ (div)-diffusion rather than fully nonlinear diffusion in the Chambolle's approach. Consequently, more efficient numerical algorithms such as time-splitting method and Fast Fourier Transform (FFT) can be implemented. Various numerical tests show that two dual algorithms are much faster and more stable than the primal gradient descent approach, and the new dual algorithm is at least as efficient as the Chambolle's algorithm but is more accurate. We demonstrate that the new method also provides a viable alternative for image restoration.
\end{abstract}

AMS subject classifications: 65N22, 65N55, 74S20, 49J40

Key words: Variational models, image segmentation, TV-Allen-Cahn type equations, dual algorithms, splitting-penalty methods, FFT.

\section{Introduction}

Image segmentation, which aims to extract interesting objects from a given image, is one fundamental task in image processing and computer vision. Among several popular

${ }^{*}$ Corresponding author. Email address: lilian@ntu.edu.sg (L.-L. Wang) 
variational models proposed for this purpose [7, 13, 20,31], the seminal Mumford and Shah model [31] can be formulated as: given an image $f$ on an open bounded domain $\Omega \subseteq \mathbb{R}^{2}$, find a partition $\Omega_{i}$ of $\Omega$ and an optimal piecewise smooth approximation $u$ of $f$ such that $u$ varies smoothly within each region $\Omega_{i}$, but rapidly and discontinuously across the boundaries $\Gamma$ of $\Omega_{i}$. The partition can be done by solving the minimization problem:

$$
\min _{u, \Gamma}\left\{E_{M S}(u, \Gamma):=\mu \int_{\Omega \backslash \Gamma}|\nabla u|^{2} d x+\frac{v}{2} \int_{\Omega}|u-f|^{2} d x+|\Gamma|\right\},
$$

where $|\Gamma|$ is the length of $\Gamma$, and $\mu, v>0$ are parameters to weight the terms in the functional. This problem involves two unknowns with different nature, and it presents a great challenge for numerical and theoretical study [2]. One may also refer to [2] for an excellent review of various attempts of approximating, relaxing or simplifying the Mumford and Shah functional as well as the numerical treatments.

Chan and Vese [13] considered a reduced version of (1.1) by assuming that the given image $f$ consists of two phases of approximately piecewise constant intensities $c_{1}$ and $c_{2}$ on the subregions $\Omega_{1}=\operatorname{inside}(\Gamma)$ and $\Omega_{2}=\operatorname{outside}(\Gamma)$, respectively. Consequently, a simplification of (1.1) leads to the Chan-Vese (CV) model:

$$
\min _{c_{1}, c_{2}, \Gamma}\left\{E_{C V}\left(c_{1}, c_{2}, \Gamma\right):=\frac{v}{2}\left(\int_{\Omega_{1}}\left|c_{1}-f\right|^{2} d x+\int_{\Omega_{2}}\left|c_{2}-f\right|^{2} d x\right)+|\Gamma|\right\},
$$

which, together with numerical implementation by the level-set method [33], has become a useful variational segmentation tool $[32,39]$.

Lie et al. $[25,26]$ proposed a piecewise constant level-set method (PCLSM) for image segmentation [40] and other interface problems [24,29], which enjoys some advantages over the classical level-set method. Different from the CV model, the PCLSM for (binary) Mumford and Shan image segmentation leads to a fully nonlinear PDE closely related to the Allen-Cahn equation for phase transition [1]. In contrast to the original Allen-Cahn equation with Laplace-diffusion, such a PDE involves a nonlinear TV-diffusion operator, so we term it as TV-Allen-Cahn model. Some algorithms have been developed and tested for the TV-Allen-Cahn model [11, 14, 24-26, 29, 34, 40]. However, most of them are based on directly evolving piecewise constant solutions (i.e., the primal variable), so oftentimes they suffer from numerical difficulties induced by the nonlinearity and non-differentiability the TV-term. In addition, the time evolution of piecewise-constantstructured solutions inevitably leads to a much severer restriction to the time step.

Motivated by the success of dual algorithms for image restoration $[8,12,19]$, we propose in this paper two efficient dual algorithms to overcome the aforementioned drawbacks of the primal approaches. The key idea is to introduce an auxiliary valuable and use a splitting-penalty method, which results in a minimization problem of Rudin-OsherFatemi (ROF) [35] type. Accordingly, this forms the base to implement efficient dual algorithms. It is worthwhile to point out that the splitting-penalty approach has been around as early as Courant [15] in 1943, and has been applied in various context of image restoration $[16,19,41,43]$. Indeed, it has proven to be an effective tool for convex optimization. 
However, we realize that for this application, the splitting-penalty technique leads to TVAllen-Cahn type models associated with "double-well" potentials different from the one (i.e., $\left(\phi^{2}-1\right)^{2}$ with $\phi$ being the phase function) in the original Allen-Cahn equation, so the penalty parameter is not necessary to be sufficiently large (slightly bigger than the parameter $\mu$ in (2.9) below), which should be in contrast to the usual penalty approach. In this paper, we first extend the Chambolle's dual algorithm [8] to such TV-Allen-Cahn type models. More importantly, we propose a new dual algorithm based on an edge-featured penalty of the dual variable, which only requires to solve a vectorial Allen-Cahn type models with linear $\nabla$ (div)-diffusion rather than fully nonlinear diffusion in the Chambolle's approach. Consequently, more efficient algorithms can be implemented on the new model such as an operator-splitting in time and FFT in space as suggested in this paper.

The rest of the paper is organized as follows. In Section 2, we briefly describe the PCLSM and the TV-Allen-Cahn equation for binary image segmentation. Then we apply a splitting-penalty method to obtain TV-Allen-Cahn type models in primal variable with various potentials. We demonstrate that the models produce desirable segmentation results as the TV-Allen-Cahn equation associated with the usual "double-well" potential. We present in Sections 3 and 4 the dual algorithms based on Chambolle's idea, and the new dual algorithm using a penalty of the dual variable along edges and implemented by an operator splitting in time and FFT in space. In Section 5, we test the algorithms on various images, and demonstrate the dual algorithms are much faster than the primal approaches, and the new algorithm slightly outperforms the dual algorithm based on Chambolle's approach in most experiments. We conclude the paper with some discussions on the applications of the new algorithm to image restoration.

\section{PCLSM and TV-Allen-Cahn type models}

We start with a brief description of the PCLSM $[25,26]$ for the minimization problem (1.2).

Different from the classical level-set method, the PCLSM identifies the contour $\Gamma$ as the discontinuity of a piecewise constant level-set function (or phase function):

$$
\phi(x)= \begin{cases}1, & x \in \operatorname{inside}(\Gamma), \\ -1, & x \in \text { outside }(\Gamma) .\end{cases}
$$

Hence, the approximation $u$ can be expressed as

$$
u=c_{1} \frac{1+\phi}{2}+c_{2} \frac{1-\phi}{2} \approx f
$$

and the length of $\Gamma$ can be characterized by the total variation of the level-set function $\phi$, defined as

$$
T V(\phi)=\int_{\Omega}|D \phi|=\sup _{p \in S} \int_{\Omega} \phi \operatorname{div} p d x
$$


where

$$
S:=\left\{\boldsymbol{p}=\left(p_{1}, p_{2}\right) \in C_{c}^{1}\left(\Omega ; \mathbb{R}^{2}\right):|\boldsymbol{p}|=\sqrt{p_{1}^{2}+p_{2}^{2}} \leq 1, \forall x \in \Omega\right\} .
$$

Consequently, the two-phase piecewise constant Mumford and Shah model (1.1) is reduced to the minimization problem (cf. [25]):

$$
\left\{\begin{array}{l}
\min _{c_{1}, c_{2}, \phi}\left\{T V(\phi)+\frac{\lambda}{2} \int_{\Omega}|u-f|^{2} d x\right\}, \quad \lambda>0, \\
\text { subject to } \phi^{2}=1
\end{array}\right.
$$

The constraint is imposed to ensure there is no vacuum and overlapping between different phases, that is, each point $x \in \Omega$ can take one and only one phase value. In [26], the constraint was treated by the augmented Lagrangian technique. Here, we use the penalty method, so the constrained minimization problem (2.5) can be approximated by (cf. [25]):

$$
\min _{c_{1}, c_{2}, \phi}\left\{L\left(c_{1}, c_{2}, \phi\right):=T V(\phi)+\frac{\lambda}{2} \int_{\Omega}|u-f|^{2} d x+\frac{\mu}{4} \int_{\Omega}\left(\phi^{2}-1\right)^{2} d x\right\}, \quad \lambda, \mu>0,
$$

where $u$ is given by (2.2). The optimality conditions lead to

$$
\begin{aligned}
& \frac{\partial L\left(c_{1}, c_{2}, \phi\right)}{\partial \phi}=-\operatorname{div}\left(\frac{\nabla \phi}{|\nabla \phi|}\right)+\lambda(u-f) \frac{\partial u}{\partial \phi}+\mu \phi\left(\phi^{2}-1\right)=0, \\
& \frac{\partial L\left(c_{1}, c_{2}, \phi\right)}{\partial c_{i}}=\int_{\Omega} u \frac{\partial u}{\partial c_{i}} d x-\int_{\Omega} f \frac{\partial u}{\partial c_{i}} d x=0, \quad i=1,2 .
\end{aligned}
$$

For fixed $\phi$, the approximation $u$ and the phase intensities $c_{1}, c_{2}$ can be updated by (2.2) and by solving the simple system $(2.7 \mathrm{~b})$, respectively. Hence, the effort needs to be devoted to the nonlinear stiff PDE (2.7a) with fixed $c_{1}$ and $c_{2}$. Most of the existing methods are based on evolving the gradient descent flow

$$
\frac{\partial \phi}{\partial t}=\operatorname{div}\left(\frac{\nabla \phi}{|\nabla \phi|}\right)-\lambda(u-f) \frac{\partial u}{\partial \phi}+\mu\left(1-\phi^{2}\right) \phi,
$$

which is closely related to the Allen-Cahn model for phase transitions [1] but with a nonlinear TV-diffusion operator rather than a linear Laplace-diffusion. To distinguish them, we term it as the TV-Allen-Cahn model. The presence of nonlinear diffusion induces some numerical difficulties, but since the TV-term has the capability to preserve discontinuities and edges, the model (2.8) might avoid mesh refinement as usually required for phase-field models $[17,36]$. Some algorithms have been developed and tested for numerical solution of (2.8), see, e.g., [11,14, 24-26, 29]. However, direct evolution of the primal variable $\phi$ might suffer from numerical difficulties due to the nonlinearity and non-differentiability of $T V(\phi)$ in (2.6). Note that the denominator of the TV-term has to be computed approximately by a regularized one:

$$
|\nabla \phi|_{\beta}=\sqrt{\phi_{x}^{2}+\phi_{y}^{2}+\beta}
$$


for some $\beta>0$. In addition, time evolution of piecewise-constant-structured solutions inevitably leads to a much severer restriction to time step. The dual algorithms have been proven to be efficient to overcome these difficulties. So far, much progress has been made in dual methods for the ROF model [8,12]. Most notably, Chambolle [8] provided a convergence analysis for the proposed dual algorithm. In addition, by introducing the auxiliary variable: $\boldsymbol{q}=\nabla \phi$ to handle the TV-term (see (3.5) below), splitting-penalty and/or augmented Lagrangian type methods have been developed in $[16,19,41,43]$ for the ROF model. Interestingly, using the augmented Lagrangian method, Tai and $\mathrm{Wu}$ [41] presented a uniform framework to derive the dual algorithms $[8,12]$ and splitting-penalty based methods $[16,19,43]$.

The Chambolle's dual algorithm essentially relies on the explicit solvability of the primal variable in terms of the dual variable, which may not be possible for the segmentation problems. Recently, Bresson et al. [6] extended the Chambolle's method to $T V-L^{1}$ minimization problem by introducing an auxiliary variable to split the $L^{1}$ data fidelity term into a quadratic one and an $L^{1}$-term of the new variable. With this treatment, the main subproblem (a $T V-L^{2}$ minimization problem) can be solved efficiently by using Chambolle's dual algorithm. Following this idea, we introduce an auxiliary $v=\phi$ to handle the nonlinearity induced by the constraint $\phi^{2}-1=0$ in (2.6), and treat the constraint $v=\phi$ by a penalty method. This leads to TV-Allen-Cahn type equations in primal variable $\phi$ obtained from (2.5) with constraints different from $\phi^{2}-1=0$ (i.e., with the potential different from the usual "double-well" in the Allen-Cahn equation). Hence, the penalty constant is not necessary to be sufficiently large in contrast to the usual penalty method.

Our starting point is to introduce an auxiliary variable and rewrite (2.6) into

$$
\min _{c_{1}, c_{2}, \phi, v}\left\{T V(\phi)+\frac{\lambda}{2} \int_{\Omega}|u-f|^{2} d x+\frac{\mu}{4} \int_{\Omega}\left(v^{2}-1\right)^{2} d x\right\}, \quad \text { subject to } v=\phi \text {. }
$$

This constrained problem can be approximated by penalizing the constraint:

$$
\min _{c_{1}, c_{2}, \phi, v}\left\{T V(\phi)+\frac{\lambda}{2} \int_{\Omega}|u-f|^{2} d x+\frac{\mu}{4} \int_{\Omega}\left(v^{2}-1\right)^{2} d x+\frac{\theta}{2} \int_{\Omega}(v-\phi)^{2} d x\right\},
$$

where the penalty parameter $\theta>0$. It is clear that if $\theta$ is sufficiently large, the problem (2.10) can accurately approximate (2.6).

However, we next show that for $\theta \geq \mu$, the model (2.10) is also robust for segmentation, which leads to TV-Allen-Cahn type equations involving a potential different from the usual "double-well" one. As for the splitting technique in [6], we seek the minimizer $\left(\phi, v, c_{1}, c_{2}\right)$ separately as follows:

(a) Fixing $v, c_{1}$ and $c_{2}$, we search for $\phi$ as a solution of

$$
\min _{\phi}\left\{T V(\phi)+\frac{\lambda}{2} \int_{\Omega}|u-f|^{2} d x+\frac{\theta}{2} \int_{\Omega}(v-\phi)^{2} d x\right\} .
$$


(b) Fixing $\phi, c_{1}$ and $c_{2}$, we search for $v$ as a solution of

$$
\min _{v}\left\{\frac{\mu}{4} \int_{\Omega}\left(v^{2}-1\right)^{2} d x+\frac{\theta}{2} \int_{\Omega}(v-\phi)^{2} d x\right\} .
$$
(2.7b).

(c) Fixing $\phi$ and $v$, the approximation $\left(c_{1}, c_{2}\right)$ of the intensities can be computed by

It is clear that the gradient descent flow of (2.11) takes the form

$$
\frac{\partial \phi}{\partial t}=\operatorname{div}\left(\frac{\nabla \phi}{|\nabla \phi|}\right)-\lambda(u-f) \frac{\partial u}{\partial \phi}+\theta(v-\phi), \quad \text { in } \Omega,
$$

and the optimality condition of (2.12) implies

$$
v^{3}+(r-1) v=r \phi, \quad \text { with } \quad r=\frac{\theta}{\mu} .
$$

Hereafter, we assume that $r \geq 1$, and find that the unique real root of (2.14) is

$$
v=G_{r}(\phi):=\sqrt[3]{\frac{r \phi}{2}+\sqrt{\frac{r^{2} \phi^{2}}{4}+\frac{(r-1)^{3}}{27}}}+\sqrt[3]{\frac{r \phi}{2}-\sqrt{\frac{r^{2} \phi^{2}}{4}+\frac{(r-1)^{3}}{27}}} .
$$

It is apparent that

$$
r\left(G_{r}(\phi)-\phi\right)=G_{r}(\phi)-G_{r}^{3}(\phi)
$$

Eq. (2.13) can be reformulated as

$$
\frac{\partial \phi}{\partial t}=\operatorname{div}\left(\frac{\nabla \phi}{|\nabla \phi|}\right)-\lambda(u-f) \frac{\partial u}{\partial \phi}+\mu\left(1-G_{r}^{2}(\phi)\right) G_{r}(\phi), \quad 1 \leq r \leq \infty .
$$

It can be regarded as a modified TV-Allen-Cahn model associated with the "double-well" potential:

$$
W_{r}(\phi)=\int_{-1}^{\phi}\left(1-G_{r}^{2}(\varphi)\right) G_{r}(\varphi) d \varphi, \quad 1 \leq r \leq \infty,
$$

in contrast to the usual "double-well" potential: $W(\phi)=\left(\phi^{2}-1\right)^{2} / 4$ corresponding to the TV-Allen-Cahn model (2.8). From a slightly different point of view, Eq. (2.17) is the gradient descent flow of (2.5) but with a different constraint to the piecewise constant level-set function $\phi$, and accordingly with the penalty term $\mu \int_{\Omega} W_{r}(\phi) d x$ in place of $\mu \int_{\Omega} W(\phi) d x$ in (2.6).

One verifies that

$$
G_{1}(\phi)=\sqrt[3]{\phi}, \quad G_{r}(\phi)=\phi+\mathcal{O}\left(r^{-\frac{3}{2}}\right), \quad r \gg 1,
$$

which implies

$$
\lim _{r \rightarrow \infty}\left\{\left(1-G_{r}^{2}(\phi)\right) G_{r}(\phi)\right\}=\left(1-\phi^{2}\right) \phi, \quad \lim _{r \rightarrow \infty} W_{r}(\phi)=W(\phi) .
$$


In addition, we find from (2.14) that

$$
\frac{d G_{r}}{d \phi}=\frac{r}{r-1+3 G_{r}^{2}(\phi)}
$$

which indicates that for $r>1, G_{r}(\phi)$ is sufficiently smooth, so is $W_{r}(\phi)$. In Fig. 1(a), we plot the graphs of several samples of $W_{r}(\phi)$ with $\phi \in[-1.5,1.5]$ and for $r=1,2,5,10,50,300$ from bottom to top by solid lines, and plot the graph of $W(\phi)$ marked by "o". We see that the "double wells" with $r>1$ are smooth, and $W_{300}(\phi)$ is almost indistinguishable from $W(\phi)=W_{\infty}(\phi)$.

To illustrate the performance of the TV-Allen-Cahn type models (2.17) in terms of the choice of $r$, we discretize it by an explicit scheme:

$$
\frac{\phi^{n+1}-\phi^{n}}{\tau}=\operatorname{div}\left(\frac{\nabla \phi^{n}}{\left|\nabla \phi^{n}\right|_{\beta}}\right)-\lambda\left(u^{n}-f\right) \frac{c_{1}^{n}-c_{2}^{n}}{2}+\mu\left(1-G_{r}^{2}\left(\phi^{n}\right)\right) G_{r}\left(\phi^{n}\right),
$$

where

$$
\left|\nabla \phi^{n}\right|_{\beta}=\sqrt{\left(\phi_{x}^{n}\right)^{2}+\left(\phi_{y}^{n}\right)^{2}+\beta}
$$

for some small $\beta>0$. The equation is equipped with the Neumann boundary condition: $\partial \phi / \partial \mathbf{n}=0$ as usual, and the divergence term is discretized by a central difference scheme as in [32] (cf. pp. 103). Note that given $\phi^{n}$, the values of $u^{n}, c_{1}^{n}$ and $c_{2}^{n}$ are evaluated by (2.2) and (2.7b). We use the annulus-square image in Fig. 1(d) as an example, and choose the parameters: $\lambda=1, \mu=200, \tau=5 e-5$ and $\beta=1 e-6$. We take the initial value of $\phi$ to be 2 inside the red box, and -2 outside (see Fig. 1(d)). To measure the convergence and accuracy, we define pixel-wise errors

$$
\begin{aligned}
& E_{\max }\left\{\phi^{n+1}, \phi^{n}\right\}=\max _{i, j}\left|\phi^{n+1}-\phi^{n}\right|, \\
& E\left\{\left|\phi^{n}\right|, 1\right\}=\max \left\{\left|\max _{i, j}\right| \phi^{n}|-1|,\left|\min _{i, j}\right| \phi^{n}|-1|\right\} .
\end{aligned}
$$

In Fig. 1(b) and 1(c), we record the history of $E_{\max }\left\{\phi^{n+1}, \phi^{n}\right\}$ and the errors $E\left\{\left|\phi^{n}\right|, 1\right\}$ to the targeted piecewise constant function (2.1). We observe a similar convergence behavior for all cases except for $r=1$. Notice that in case of $r=1$, the potential $W_{r}(\phi)$ is not differentiable at $\phi=0$. We plot in Fig. 1(f) the profile of the phase function $\phi$ at 180 steps, which shows a good approximation of the piecewise constant level-set as expected. In Fig. 1(e), we depict the boundaries after segmentation, which indicates a quite accurate detection for $r=10$ at 180 steps.

As with ROF model based image restoration, the primal approach is born with slow convergence and severe restriction on time stepping size, although some improvement can be made by using, e.g., the AOS scheme [27, 28, 44]. Moreover, in this context, it is also quite sensitive to the choice of $\phi^{0}$ (refer to the second numerical example in Section 5). We next introduce two dual algorithms to overcome these shortcomings. 


\section{Dual algorithm based on Chambolle [8]}

Now, we are in a position to present the fast dual algorithms for the minimization problem (2.10). Since the subproblem (2.12) can be solved analytically, we only need to focus on the subproblem (2.11).

As $u$ is a linear function of $\phi$, the subproblem (2.11) with fixed $c_{1}, c_{2}$ and $v$ can be solved by using Chambolle's dual algorithm. More precisely, the level-set function $\phi$ is computed by

$$
\phi=\mathcal{C}\left(p, v, c_{1}, c_{2}\right):=\frac{1}{\lambda\left(c_{1}-c_{2}\right)^{2} / 4+\theta}\left(\frac{\lambda\left(c_{1}-c_{2}\right)}{2} f-\operatorname{div} p+\theta v-\lambda \frac{c_{1}^{2}-c_{2}^{2}}{4}\right),
$$

and the dual variable $\boldsymbol{p}=\left(p_{1}, p_{2}\right)$ satisfies

$$
\nabla \boldsymbol{H}-|\nabla \boldsymbol{H}| \boldsymbol{p}=0
$$

where

$$
\boldsymbol{H}:=\boldsymbol{H}\left(\boldsymbol{p}, v, c_{1}, c_{2}\right)=\nabla\left(\operatorname{div} \boldsymbol{p}-\frac{\lambda\left(c_{1}-c_{2}\right)}{2} f-\theta v\right) .
$$

For fixed $c_{1}, c_{2}$ and $v$, the problems (3.2)-(3.3) can be solved by the iteration with $p^{0}=0$ :

$$
\boldsymbol{p}^{n+1}=\frac{\boldsymbol{p}^{n}+\tau \boldsymbol{H}\left(\boldsymbol{p}^{n}, v, c_{1}, c_{2}\right)}{1+\tau\left|\boldsymbol{H}\left(\boldsymbol{p}^{n}, v, c_{1}, c_{2}\right)\right|^{\prime}}, \quad n=0,1, \cdots .
$$

It is anticipated that the iteration converges for $0<\tau \leq 1 / 8$ as with the original algorithm for ROF model in [10], and might be sped up by using the technique in [9]. In addition, one may follow [10] to derive (3.2) by using the Karush-Kuhn-Tucker conditions for inequality constrained optimization problems. However, it can be derived by a much simper argument as in [41]. For the interested readers' reference, we briefly sketch the derivation followed from [41] below. We first convert the subproblem (2.11) into the following constrained minimization:

$$
\left\{\begin{array}{l}
\min _{\boldsymbol{q}, \phi}\left\{\int_{\Omega}|\boldsymbol{q}| d x+\frac{\lambda}{2} \int_{\Omega}|u-f|^{2} d x+\frac{\theta}{2} \int_{\Omega}(v-\phi)^{2} d x\right\}, \\
\text { subject to } \boldsymbol{q}=\nabla \phi .
\end{array}\right.
$$

Using the notion of augmented Lagrangian method, we obtain

$$
\begin{aligned}
\min _{\phi, \boldsymbol{q}} \max _{\boldsymbol{p}}\{ & \mathcal{F}(\phi, \boldsymbol{q}, \boldsymbol{p}):=\int_{\Omega}|\boldsymbol{q}| d \boldsymbol{x}+\frac{\lambda}{2} \int_{\Omega}|u-f|^{2} d \boldsymbol{x}+\frac{\theta}{2} \int_{\Omega}(v-\phi)^{2} d \boldsymbol{x} \\
& \left.+\int_{\Omega} \boldsymbol{p} \cdot(\boldsymbol{q}-\nabla \phi) d \boldsymbol{x}+\frac{\gamma}{2} \int_{\Omega}|\boldsymbol{q}-\nabla \phi|^{2} d \boldsymbol{x}\right\}, \quad \lambda, \gamma, \theta>0,
\end{aligned}
$$

where $\boldsymbol{p}=\left(p_{1}, p_{2}\right)$ is the Lagrange multiplier. By the optimality conditions, 


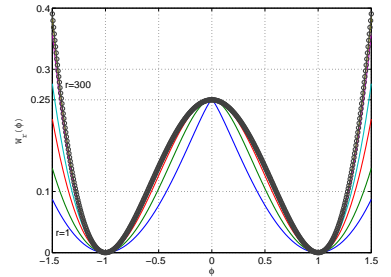

(a) Samples of $W_{r}(\phi)$ and $W(\phi)$

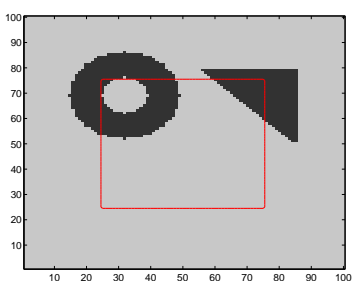

(d) Given image and initial value

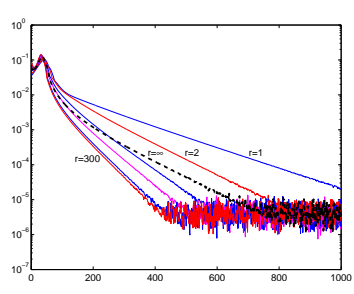

(b) Error: $E_{\max }\left\{\phi^{n+1}, \phi^{n}\right\}$

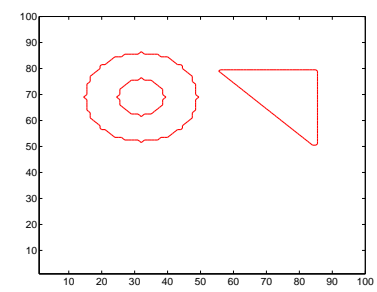

(e) Segmented $\Gamma(r=10)$

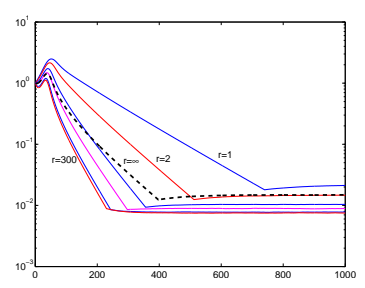

(c) Error: $E\left\{\left|\phi^{n}\right|, 1\right\}$

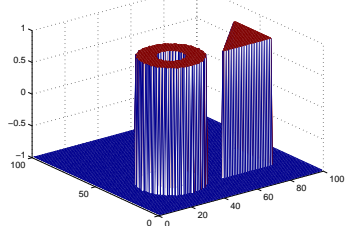

(f) $\phi$ at convergence $(r=10)$

Figure 1: Profiles of the "double-well" potentials, convergence behavior (from right to left: $r=1,2, \infty, 5,10$, $50,300)$, and segmented results obtained by $(2.21)$.

$$
\begin{aligned}
& \frac{\partial \mathcal{F}(\phi, \boldsymbol{q}, \boldsymbol{p})}{\partial \phi}=\lambda(u-f) \frac{\partial u}{\partial \phi}-\theta(v-\phi)+\operatorname{div} \boldsymbol{p}+\gamma \operatorname{div}(\boldsymbol{q}-\nabla \phi)=0, \\
& \frac{\partial \mathcal{F}(\phi, \boldsymbol{q}, \boldsymbol{p})}{\partial \boldsymbol{q}}=\frac{\boldsymbol{q}}{|\boldsymbol{q}|}+\boldsymbol{p}+\gamma(\boldsymbol{q}-\nabla \phi)=0, \\
& \frac{\partial \mathcal{F}(\phi, \boldsymbol{q}, \boldsymbol{p})}{\partial \boldsymbol{p}}=\boldsymbol{q}-\nabla \phi=0 .
\end{aligned}
$$

Eq. (3.2) follows from (3.7b) and (3.7c). Moreover, we find from (3.7a) and (3.7c) that

$$
\lambda(u-f) \frac{\partial u}{\partial \phi}-\theta(v-\phi)+\operatorname{div} p=0
$$

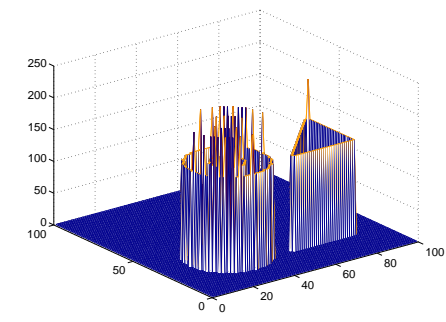

(a) $|\nabla f|$

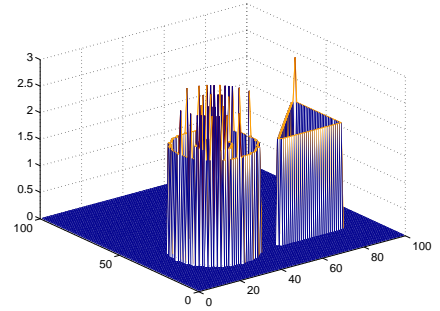

(b) $|\nabla \phi|=|\boldsymbol{H}| / d$

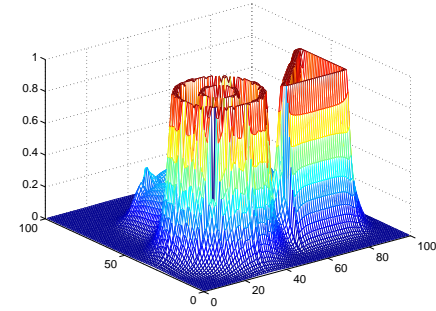

(c) $|p|$

Figure 2: Profiles of $|\nabla f|,|\nabla \phi|$ and $|p|$ obtained by Algorithm 3.1 with $\lambda=1, \mu=1 e 3, r=2, \tau=0.1$ for $N=100$ steps. Note: (i) the error $E_{\max }\left[\phi^{N}, \phi^{N-1}\right]=2.1 e-7$, and (ii) as in Fig. $1(\mathrm{f}), \phi$ converges to (2.1), so we have approximately $|\nabla \phi| \leq 2 \sqrt{2}$. 
In view of (2.2), we can express $\phi$ in terms of the dual variable $p$ and obtain (3.1). Finally, scaling out the constant factor

$$
d:=d\left(c_{1}, c_{2}\right)=\frac{\lambda}{4}\left(c_{1}-c_{2}\right)^{2}+\theta,
$$

and applying a semi-implicit scheme to the corresponding gradient descent flow (cf. [8]), we obtain the scheme (3.4).

As usual, a homogeneous Dirichlet boundary condition: $\left.p\right|_{\partial \Omega}=0$ can be imposed. Here, we use a periodic boundary condition for comparison with the new dual algorithm in the next section. We also note that $\boldsymbol{H}$ can be discretized by finite difference as in [8].

We summarize the dual algorithm for (2.11)-(2.12) as Algorithm 3.1 below.

Algorithm 3.1:

1. Initialization: choose $\phi^{0}$ and set $p^{0}=0$.

2. For $n=0,1, \cdots$,

(i) Compute $\left(c_{1}^{n}, c_{2}^{n}\right)$ by solving the simple system (2.7b) with $\phi=\phi^{n}$.

(ii) Compute $u^{n}$ by

$$
u^{n}=\frac{1+\phi^{n}}{2} c_{1}^{n}+\frac{1-\phi^{n}}{2} c_{2}^{n} .
$$

(iii) Compute $v^{n}$ by $v^{n}=G_{r}\left(\phi^{n}\right)$ in (2.15).

(iv) Compute $p^{n+1}$ by (3.4):

$$
p^{n+1}=\frac{\boldsymbol{p}^{n}+\tau \boldsymbol{H}\left(\boldsymbol{p}^{n}, v^{n}, c_{1}^{n}, c_{2}^{n}\right)}{1+\tau\left|\boldsymbol{H}\left(\boldsymbol{p}^{n}, v^{n}, c_{1}^{n}, c_{2}^{n}\right)\right|} .
$$

(v) Compute $\phi^{n+1}$ by (3.1):

$$
\phi^{n+1}=\mathcal{C}\left(p^{n+1}, v^{n}, c_{1}^{n}, c_{2}^{n}\right) .
$$

3. Goto 2 till some stopping rule (e.g, $E_{\max }\left[\phi^{n+1}, \phi^{n}\right]<\varepsilon$, for some $\varepsilon>0$ ) is met.

\section{Dual algorithm based on edge-featured penalty and FFT}

The previous dual approach for (2.11) requires to solve the nonlinear PDE (3.2), whose leading operator is highly nonlinear. In this section, we propose a new model for the dual variable with a linear leading operator, which allows for the use of more efficient numerical algorithms. The key idea is based on an edge-featured penalty of the inequality constraint $|p| \leq 1$ in the definition of the TV-norm. It is important to observe that (cf. [10])

$$
|\boldsymbol{p}|=1, \quad \text { if } \boldsymbol{H} \neq 0 ; \quad|\boldsymbol{p}|<1, \quad \text { if } \boldsymbol{H}=0 .
$$


Moreover, we find from (3.1), (3.3) and (3.9) that $\boldsymbol{H}=d \nabla \phi$. As $\phi$ is designed to characterize the piecewise constant structures of the given image $f$, we have $|\boldsymbol{H}|=\mathcal{O}(|\nabla \phi|)=\mathcal{O}(|\nabla f|)$. Indeed, their profiles in Fig. 2 justify this observation. Consequently, we treat the pointwise inequality constraint by penalizing $|p|=1$ strongly near sharp edges, and very weakly in the homogeneous regions. More precisely, we propose to approximate the subproblem (2.11) by the following minimization problem:

$$
\min _{\phi} \max _{p}\left\{\int_{\Omega} \phi \operatorname{div} p d x+\frac{\lambda}{2} \int_{\Omega}|u-f|^{2} d x+\frac{\theta}{2} \int_{\Omega}(v-\phi)^{2} d x-\frac{1}{4} \int_{\Omega} g(|\nabla f|)\left(|p|^{2}-1\right)^{2} d x\right\},
$$

where $g(s)$ is a smooth function of $s \in[0, \infty)$, such that $g(0)=0$ and $g(s) \gg 1$ for $s \gg 1$. In this paper, we take

$$
g(s)=\alpha \ln (1+s), \quad s \geq 0,
$$

where $\alpha$ is a positive constant.

The optimality condition with respect to $\phi$ yields $\phi=\mathcal{C}\left(p, v, c_{1}, c_{2}\right)$ defined in (3.1), and the one with respect to $p$ gives

$$
-\nabla \phi+g(|\nabla f|)\left(1-|\boldsymbol{p}|^{2}\right) \boldsymbol{p}=0
$$

In view of (3.1) and (3.3), its gradient descent form is

$$
\frac{\partial \boldsymbol{p}}{\partial t}=d^{-1} \boldsymbol{H}\left(\boldsymbol{p}, v, c_{1}, c_{2}\right)+g(|\nabla f|)\left(1-|\boldsymbol{p}|^{2}\right) \boldsymbol{p},
$$

where $\boldsymbol{H}$ and $\boldsymbol{d}$ are given in (3.3) and (3.9), respectively. We can view (4.5) as a vectorial Allen-Cahn type model with $\nabla$ (div)-diffusion.

To resolve (4.5) efficiently, we adopt a splitting scheme (or operator splitting, cf. [30, 37]), and solve the following two subproblems consecutively and recursively at each time step:

$$
\begin{aligned}
& \frac{\partial p}{\partial t}=d^{-1} \boldsymbol{H}\left(\boldsymbol{p}, v, c_{1}, c_{2}\right), \\
& \frac{\partial p}{\partial t}=g(|\nabla f|)\left(1-|\boldsymbol{p}|^{2}\right) \boldsymbol{p} .
\end{aligned}
$$

Let $p^{n}$ be the approximation of $p$ at $t_{n}=n \Delta t$. Formally, the second-order splitting scheme can be carried out as follows:

(a) Solve Eq. (4.6b) for $t \in\left(t_{n}, t_{n}+\Delta t / 2\right)$ :

$$
\tilde{\boldsymbol{p}}=\boldsymbol{p}^{n} \exp \left(g(|\nabla f|)\left(1-\left|\boldsymbol{p}^{n}\right|^{2}\right) \frac{\Delta t}{2}\right) .
$$

(b) Solve Eq. (4.6a) for $t \in\left(t_{n}, t_{n}+\Delta t\right)$ by using the Crank-Nicolson type scheme (see, e.g., [38]):

$$
\frac{\hat{\boldsymbol{p}}-\tilde{\boldsymbol{p}}}{\Delta t}=\frac{1}{2 d}\left(\boldsymbol{H}\left(\hat{\boldsymbol{p}}, v, c_{1}, c_{2}\right)+\boldsymbol{H}\left(\tilde{\boldsymbol{p}}, v, c_{1}, c_{2}\right)\right) .
$$


(c) Find $p^{n+1}$ by solving (4.6b) for $t \in\left(t_{n}+\Delta t / 2, t_{n+1}\right)$ :

$$
\boldsymbol{p}^{n+1}=\hat{\boldsymbol{p}} \exp \left(g(|\nabla f|)\left(1-|\hat{\boldsymbol{p}}|^{2}\right) \frac{\Delta t}{2}\right) .
$$

It is seen that with a linearization, Eq. (4.6b) becomes a simple ordinary differential equation. By imposing a periodic boundary condition for $p$ (see [41,43] for similar imposition), the linear equation (4.8) can be solved efficiently by using the Fourier-Galerkin method and FFT. One may refer to [42] for the implementation of FFT in Matlab.

We replace Step (iv) in Algorithm 3.1 by the splitting-FFT scheme and summarize the full algorithm for the minimization problem (2.10) in Algorithm 4.1 below.

Algorithm 4.1

Just replace Step (iv) in Algorithm 3.1 by the splitting-FFT scheme for (4.5), and remain the other steps. More precisely,

(iv) Compute $p^{n+1}$ by

$$
\left\{\begin{array}{l}
\tilde{\boldsymbol{p}}=\exp \left(g(|\nabla f|)\left(1-\left|\boldsymbol{p}^{n}\right|^{2}\right) \frac{\Delta t}{2}\right) \boldsymbol{p}^{n} \\
-\frac{\Delta t}{2 d^{n}} \nabla(\operatorname{div} \hat{\boldsymbol{p}})+\hat{\boldsymbol{p}}=\tilde{\boldsymbol{p}}+\frac{\Delta t}{2 d^{n}} \nabla(\operatorname{div} \tilde{\boldsymbol{p}})-\frac{\Delta t}{d^{n}} s^{n} \\
\boldsymbol{p}^{n+1}=\exp \left(g(|\nabla f|)\left(1-|\hat{\boldsymbol{p}}|^{2}\right) \frac{\Delta t}{2}\right) \hat{\boldsymbol{p}}
\end{array}\right.
$$

where

$$
d^{n}=\frac{\lambda}{4}\left(c_{1}^{n}-c_{2}^{n}\right)^{2}+\theta, \quad s^{n}=\frac{\lambda\left(c_{1}^{n}-c_{2}^{n}\right)}{2} \nabla f+\theta \nabla v^{n} .
$$

The second equation is solved by the Fourier-Galerkin method with FFT.

We point out that due to the use of FFT, this algorithm is competitive to the primal approach and the dual Algorithm 3.1 in terms of computational complexity.

\section{Numerical results}

The preliminary experiments in Section 2 have shown that the TV-Allen-Cahn type models (2.17) with various choices of $r$ (i.e., "double-well" potentials) are robust for binary image segmentation. In this section, we further test the models on several typical images with an emphasis on comparing the performance of the foregoing three algorithms, that is, the primal approach (2.21), the dual Algorithm 3.1 and the dual Algorithm 4.1. We assess their performance by examining the rate of convergence, decay of numerical energy and quality of segmentation.

In the first experiment, we still test the $100 \times 100$ annulus-triangle image with the same initial value (cf. Fig. 1(d)), and take the parameters: $\lambda=1, \mu=500, r=2$ in (2.17), $\alpha=20$ in 
(4.3) and $\beta=1 e-6$ in (2.21). To set up a relatively fair rule for comparison, we take the artificial time-marching step size $\tau$ or $\Delta t$ to be comparable with values $1 e-3,1 e-4,5 e-5$ and $1 e-5$ for the primal approach and Algorithm 4.1. As the factor $d(\approx 2500)$ in $(3.9)$ is scaled out in Algorithm 3.1 (cf. (3.4)), there holds the relation between the time steps of two algorithms: $\tau \approx d \Delta t$. Accordingly, we choose $\tau=2.5,0.25,0.125$ and 0.025 in Algorithm 3.1.

We quantify the comparison by recording in Fig. 3: (i) maximum pixel-wise errors between two consecutive steps: $E_{\max }\left\{\phi^{n+1}, \phi^{n}\right\}$; (ii) history of converging to the piecewise constant function (2.1) by examining $E\left\{\left|\phi^{n}\right|, 1\right\}$ (cf. (2.22)); and (iii) decay of numerical energy of the cost functional in (2.10).

We visualize from Fig. 3 that two dual algorithms converge much faster than the primal approach. In other words, the phase function $\phi$ evolves more rapidly to the targeted piecewise constant level-set function by dual methods. Moreover, we see that Algorithm 4.1 produces relatively accurate approximations than the other two methods. We remark that (i) for $\tau=1 e-3$, the primal approach fails to converge due to the restriction of the CFL condition; (ii) the dual Algorithm 3.1 still converges with a choice of $\tau$ bigger than the critical value $1 / 8$ (cf. [8] for the ROF model). However, the convergence rate and accuracy deteriorate, when $\tau>1 / 4$ (note: the choice of $\tau \leq 1 / 4$ is shown to be numerically stable in [8]).

Next, we test an image used in $[3,6]$ for discussing the global minimization issue of the variational segmentation using CV-model and the classical level-set method. As shown in [3], the minimization algorithms based on some commonly-used explicit or implicit time evolution of the gradient descent flow often get stuck with a local minimizer, and therefore leads to a rather unsatisfactory segmentation of the narrow L-band in Fig. 4(a). In this test, we choose the parameters and time steps in two dual algorithms as $\lambda=1, \mu=$ $200, r=2, \Delta t=1 e-5, \tau=1 / 8$ and $\alpha=1 e 3$, and take two typical initial values (cf. Fig. 4(a) and notice that the one at the bottom-left corner is similar to that in [6]).

We observe from Fig. 4 that the numerical phase function $\phi$ evolved by the dual algorithms converges very fast to the piecewise constant level-set function even with initial conditions far from the targets. Unfortunately, the primal approach (2.21) does not converge even for various attempts of different sets of parameters and time steps. However, the dual algorithms are very reliable and produce accurate segmentation with around 130 steps (see Fig. 4(b)). As with the previous example, two dual algorithms enjoy almost the same convergence behavior, but Algorithm 4.1 seems to be slightly accurate. Hence, they are indistinguishable when we plot the boundaries of the segmented image, and the profiles of $\phi$. In fact, similar performance is observed in the following tests involving relatively complicated images (see Fig. 5 for the newspaper segmentation, and Fig. 6 for the two-cell image with noise).

In particular, we point out that the noisy two-cell image of the same size in Fig. 6(a) was tested in [21], where the term $\int_{\Omega}\left(|\nabla \phi|^{2}-1\right)^{2} d x$ was added to penalize the classical level-set function so as to eliminate the re-initialization process. The explicit evolution of the primal variable in [21] required at least 800 iterations to reach a meaningful segmen- 


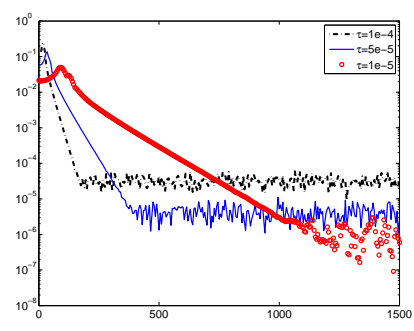

(a) Primal: $E_{\max }\left\{\phi^{n+1}, \phi^{n}\right\}$

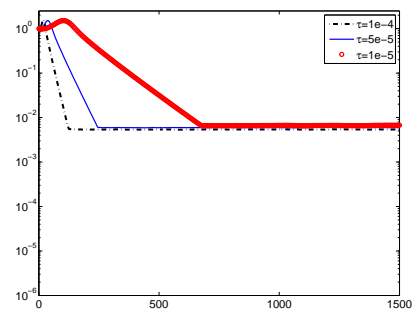

(d) Primal: $E\left\{\left|\phi^{n}\right|, 1\right\}$

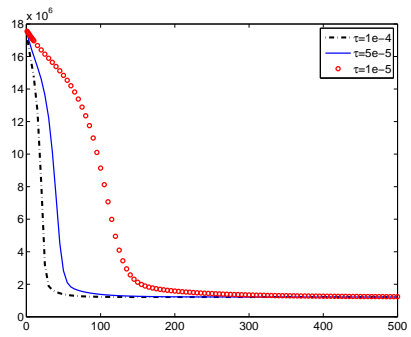

(g) Primal: decay of energy

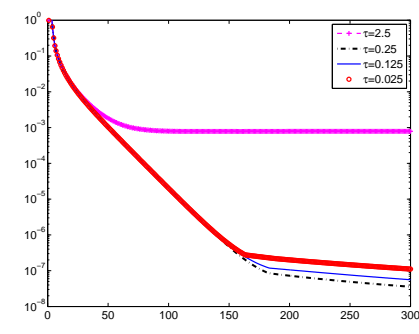

(b) Algorithm 3.1: $E_{\max }\left\{\phi^{n+1}, \phi^{n}\right\}$

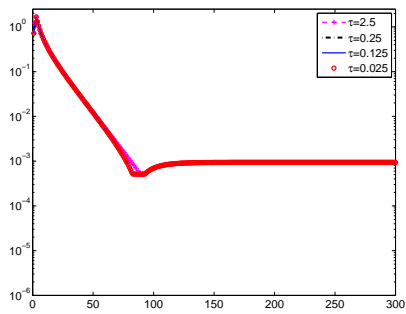

(e) Algorithm 3.1: $E\left\{\left|\phi^{n}\right|, 1\right\}$

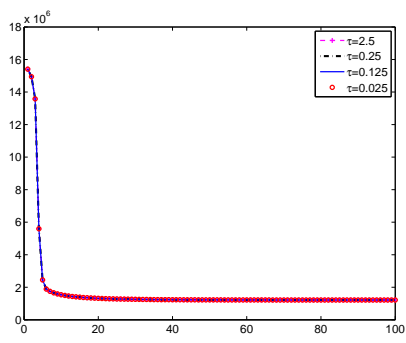

(h) Algorithm 3.1: decay of energy

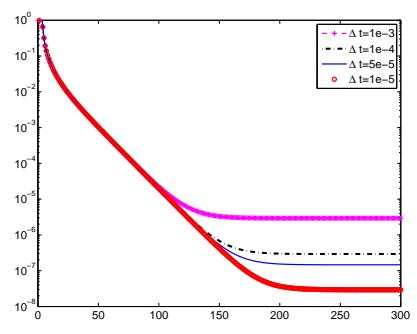

(c) Algorithm 4.1: $E_{\max }\left\{\phi^{n+1}, \phi^{n}\right\}$

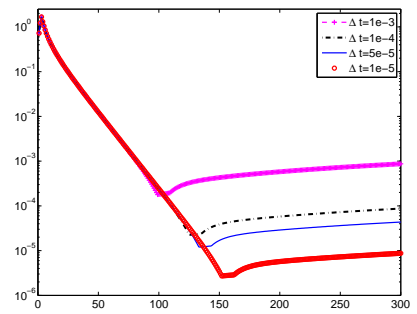

(f) Algorithm 4.1: $E\left\{\left|\phi^{n}\right|, 1\right\}$

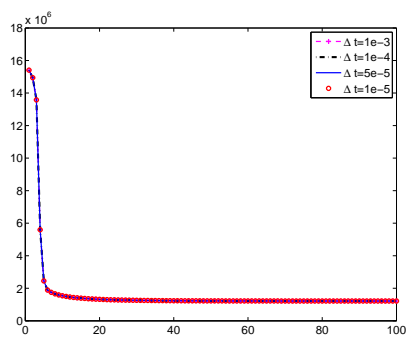

(i) Algorithm 4.1: decay of energy

Figure 3: Comparison of the convergence rate with respect to the time-marching step $n$ for three different time step sizes. Row 1: the maximum point-wise errors between two consecutive steps: $E_{\max }\left\{\phi^{n+1}, \phi^{n}\right\}$. Row 2: the convergence rate of $\left|\phi^{n}\right|$ to 1 measured by $E\left\{\left|\phi^{n}\right|, 1\right\}$. Row 3: Decay of numerical energy of the cost functional in (2.10) of three algorithms.

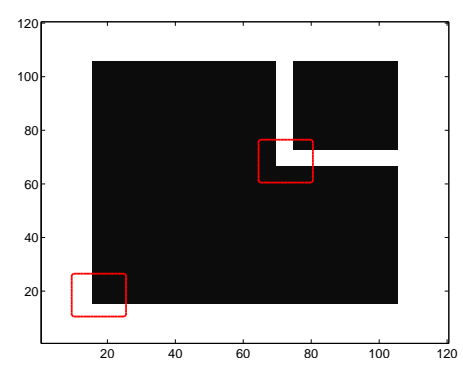

(a) Given image and initial $\phi$

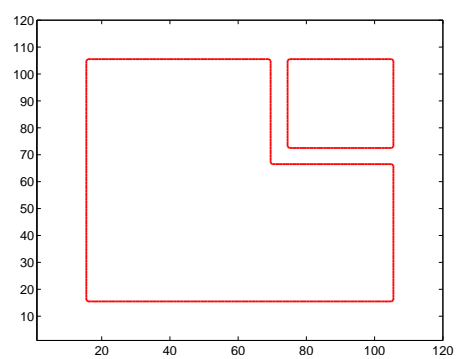

(b) Segmented $\Gamma$ (i.e., $\phi=0$ )

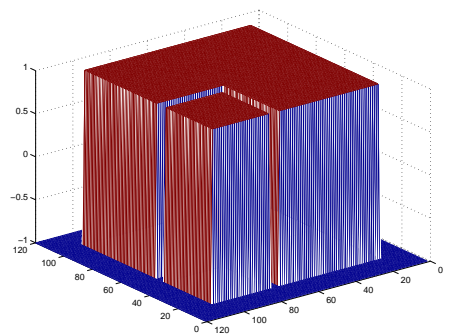

(c) $\phi$ at convergence

Figure 4: Segmentation by Algorithms 3.1 and 4.1 with two different choices of initial values (note: the primal approach fails to converge for both options): (a) given image $f$ and two samples of initial values; (b) segmented boundary $\Gamma$ i.e., $\phi=0$ at 130 steps; (c) profile of $\phi$ at 130 steps. 


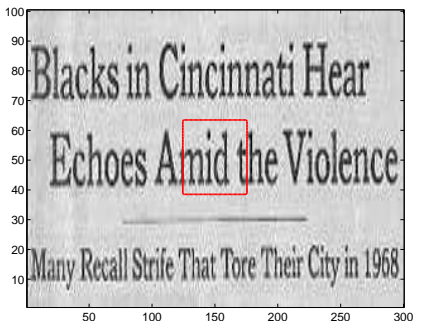

(a) Given image and initial value

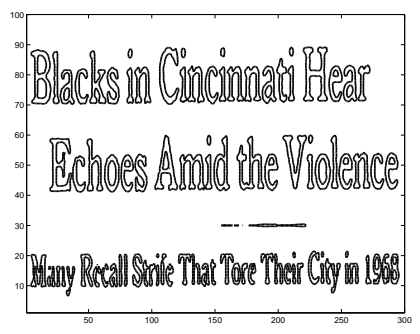

(b) Segmented $\Gamma$ (i.e., $\phi=0$ )

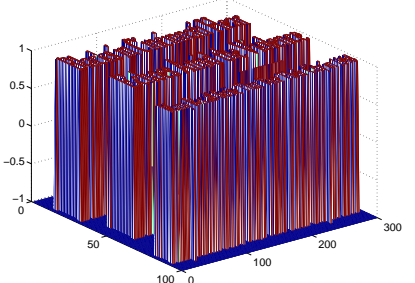

(c) $\phi$ at convergence.

Figure 5: Segmentation by Algorithms 3.1 and 4.1 with $\lambda=1, \mu=1 e 3, r=10, \alpha=1 e 3, \tau=1 / 8$ and $\Delta t=1 e-5$ : (a) given image $f$ and $\phi^{0}$; (b) segmented boundary $\Gamma$ i.e., $\phi=0$ at 110 steps; (c) profile of $\phi$ at 110 steps.

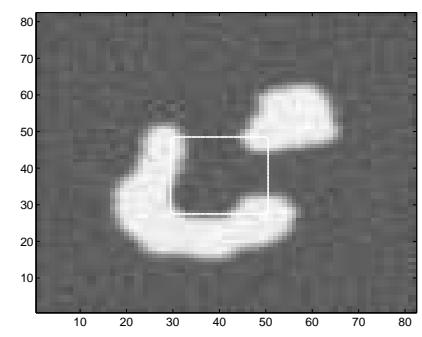

(a) Given image $f$ and $\phi^{0}$

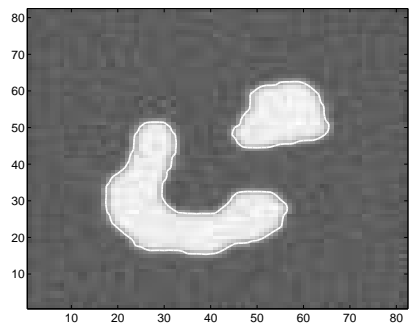

(b) $u$ at 80 steps

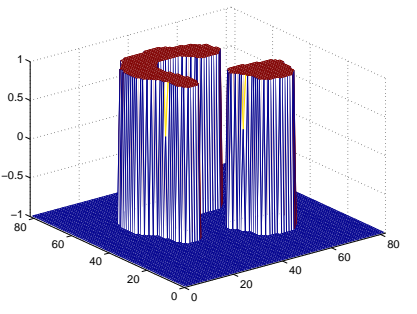

(c) $\phi$ at 80 steps

Figure 6: Segmentation by Algorithms 3.1 and 4.1 with the same parameters as in Fig. 5: (a) given image $f$ and $\phi^{0}$; (b) segmented image with marked $\Gamma$ at 80 steps; (c) Profile of $\phi$ at 80 steps.

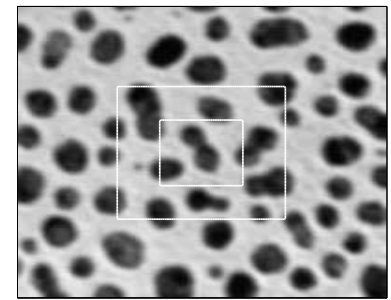

(a) Given image $f$ and initial value

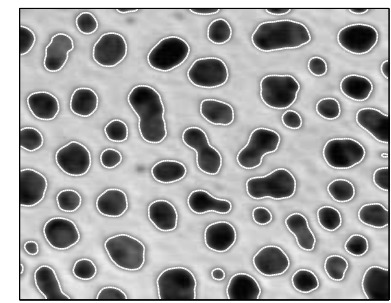

(b) $u$ at 35 steps

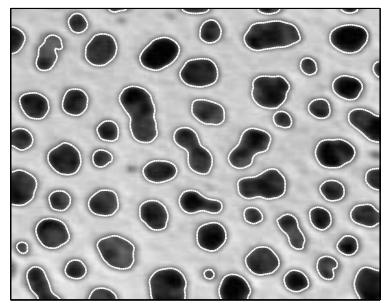

(c) $u$ at 40 steps

Figure 7: Segmentation by Algorithms 4.1 with $\lambda=1, \mu=1 e 3, \tau=1 e-5, \alpha=2 e 3$ and $r=10$ : (a) given image and two different initial conditions; (b) segmented image $u$ for the initial value given by bigger box at 35 steps; (c) segmented image $u$ for the initial value given by smaller box at 40 steps. Note: the errors $E\left\{\left|\phi^{n}\right|, 1\right\}$ for (b) and $(\mathrm{c})$ are $1.16 e-1$ and $5.61 e-2$, respectively.

tation. However, both dual algorithms provide accurate segmentation with 80 iterations, as shown in Fig. 6.

Finally, we use Algorithm 4.1 to segment the image in Fig. 7(a) with quite a number of cells of different sizes but without noise. For two choices of initial values, the dual Algorithm 4.1 produces very fast and reliable segmentation. We see that though at around 40 steps, the errors $E\left\{\left|\phi^{n}\right|, 1\right\}$ are of accuracy about one or two significant digits, the segmentation of the set $\Gamma$ is quite satisfactory. 


\section{Extensions and discussions}

It is seen that Algorithm 4.1 is very efficient for the subproblem (2.11). It only requires to solve a vectorial Allen-Cahn type equation with leading linear differential operator, so more efficient algorithms can be implemented. The previous study and numerical results demonstrate that it provides a viable alternative to the Chambolle's algorithm [8]. We next show that it is also competitive for solving the ROF model.

As the Chamoblle's approach was originally proposed for the ROF model [35], it is desirable to compare the performance of both dual algorithms for image restoration. Given a noisy image $f$ defined on an open bounded domain $\Omega \subseteq \mathbb{R}^{2}$, the ROF model restores $f$ by solving the minimization problem:

$$
\min _{u}\left\{T V(u)+\frac{1}{2 \delta} \int_{\Omega}|u-f|^{2} d x\right\}, \quad \delta>0 .
$$

Like (2.11) and (4.2), a key idea to minimize (6.1) is to consider the following new minimization problem:

$$
\min _{u} \max _{p}\left\{\int_{\Omega} u \operatorname{div} p d x+\frac{1}{2 \delta} \int_{\Omega}|u-f|^{2} d x-\frac{1}{4} \int_{\Omega} g(|\nabla f|)\left(|p|^{2}-1\right)^{2} d x\right\} .
$$

Consequently, the optimality conditions lead to

$$
u=f-\delta \operatorname{div} p
$$

and

$$
\nabla(\delta \operatorname{div} \boldsymbol{p}-f)+g(|\nabla f|)\left(1-|\boldsymbol{p}|^{2}\right) \boldsymbol{p}=0 .
$$

Like (4.5), we consider the gradient flow

$$
\frac{\partial \boldsymbol{p}}{\partial t}=\delta \boldsymbol{H}(\boldsymbol{p})+g(|\nabla f|)\left(1-|\boldsymbol{p}|^{2}\right) \boldsymbol{p}
$$

where $\boldsymbol{H}(\boldsymbol{p})=\nabla(\operatorname{div} \boldsymbol{p})-\nabla f / \delta$. Similarly, it can be solved efficiently by using a timesplitting scheme and FFT as in (4.6a)-(4.9).

Recall that in [8], the ROF model (6.1) is solved by the iteration:

$$
\boldsymbol{p}^{n+1}=\frac{\boldsymbol{p}^{n}+\tau \boldsymbol{H}\left(\boldsymbol{p}^{n}\right)}{1+\tau\left|\boldsymbol{H}\left(\boldsymbol{p}^{n}\right)\right|}, \quad n=0,1, \cdots,
$$

and $u$ is updated by (6.3).

We test two dual algorithms on lena-512 $\times 512$, and as usual the peak signal-to-noise (PSNR) is used as a criteria for the quality of restoration:

$$
\mathrm{PSNR}=10 \log _{10} \frac{255^{2}}{\frac{1}{m n} \sum_{i, j}\left(u_{i, j}-f_{i, j}\right)^{2}},
$$




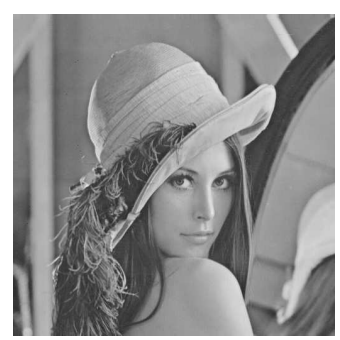

(a) Original image

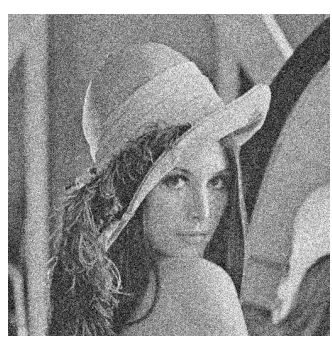

(b) Noisy image $f$

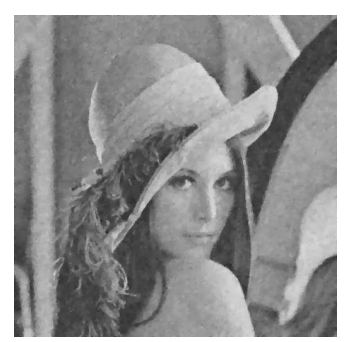

(c) $u$-Chambolle

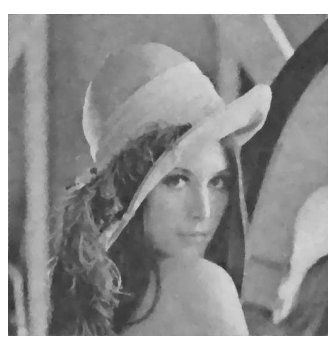

(d) $u$-new algorithm

Figure 8: Image restoration based on the ROF model with solutions by the Chambolle's dual approach and the proposed new algorithm: (a) Original image: lena- $512 \times 512$; (b) Noisy image generated by the normal imnoise function in Matlab with "Gaussian", zero mean and variance 0.02; (c)-(d): Restored images obtained by Chambolle's method and the new dual algorithm with $\delta=30, \tau=1 / 8, \Delta t=1 e-2$ and $g(s)=0.6 \sqrt[4]{s}$. The iteration is stopped by $E_{l^{2}}\left(u^{n+1}, u^{n}\right) \leq 1 e-3$. The iteration numbers and PSNR for (c)-Chambolle: 101 and 29.21, and for (d)-new algorithm: 89 and 29.16, respectively.

where $m \times n$ is the size of the image. Moreover, we use the relative dynamic error:

$$
E_{l^{2}}\left(u^{n+1}, u^{n}\right):=\frac{\left\|u^{n+1}-u^{n}\right\|_{l^{2}}}{\left\|u^{n+1}\right\|_{l^{2}}}<\epsilon,
$$

for a prescribed tolerance $\epsilon$, as the stopping rule. We see from Fig. 8 and the caption therein that with the same error tolerance, Algorithm 4.1 converges relatively faster with almost the same PSNR values. Such a behavior is consistent with the numerical experiments for image segmentation in the previous section.

Much of our previous study has been focused on the binary image segmentation. It is interesting to discuss the extension of the algorithms to multi-phase segmentation by using the Mumford and Shah model and PSCLM in [26], but it is much more challenging and involved. We leave this topic to a future work. Indeed, it is worthwhile to mention some recent advances in multi-phase segmentation in terms of developing new algorithms and using more general models such as the Potts-type models (see, e.g., $[4,5,18,22,23]$ and the references therein).

\section{Acknowledgments}

This research is supported by Singapore AcRF Tier 1 Grant RG58/08, Singapore MOE Grant T207B2202 and Singapore NRF2007IDM-IDM002-010.

\section{References}

[1] S. M. Allen and J. W. Cahn, A microscope theory for antiphase boundary motion and its application to antiphase domain coarsening, Acta. Metall., 27(6) (1979), 1085-1095.

[2] G. Aubert and P. Kornprobst, Mathematical Problems in Image Processing: Partial Differential Equations and the Calculus of Variations, Springer-Verlag, New York Inc, 2006. 
[3] N. Badshah and K. Chen, Multigrid method for the Chan-Vese model in variational segmentation, Commun. Comput. Phys., 4(2) (2008), 294-316.

[4] N. Badshah and K. Chen, Image selective segmentation under geometrical constraints using an active contour approach, Commun. Comput. Phys., 7(4) (2010), 759-778.

[5] E. Bae, J. Yuan and X. C. Tai, Global minimization for continuous multiphase partitioning problems using a dual approach, UCLA, CAM-Report (09-75), 2009.

[6] X. Bresson, S. Esedoglu, P. Vandergheynst, J. P. Thiran, and S. Osher, Fast global minimization of the active contour/snake model, J. Math. Imaging. Vis., 28(2) (2007), 151-167.

[7] V. Caselles, R. Kimmel, and G. Sapiro, Geodesic active contours, Int. J. Comput. Vis., 22(1) (1997), 61-79.

[8] A. Chambolle, An algorithm for total variation minimization and applications, J. Math. Imaging. Vis., 20(1) (2004), 89-97.

[9] A. Chambolle, Total variation minimization and a class of binary MRF models, In Energy Minimization Methods in Computer Vision and Pattern Recognition, pages 136-152, Springer, 2005.

[10] A. Chambolle and P. L. Lions, Image recovery via total variation minimization and related problems, Numer. Math., 76(2) (1997), 167-188.

[11] T. F. Chan, K. Chen, and X. C. Tai, Nonlinear multilevel schemes for solving the total variation image minimization problem, In Image Processing Based on Partial Differential Equations, Series of Math Vision, pages 265-288, Springer, Berlin, 2007.

[12] T. F. Chan, G. H. Golub, and P. Mulet, A nonlinear primal-dual method for total variationbased image restoration, SIAM J. Sci. Comput., 20(6) (1999), 1964-1977.

[13] T. F. Chan and L. A. Vese, Active contours without edges, IEEE Trans. Image. Proc., 10(2) (2001), 266-277.

[14] O. Christiansen and X. C. Tai, Fast implementation of piecewise constant level set methods, In Image Processing Based on Partial Differential Equations, Series of Math Vision, pages 289-308, Springer, Berlin, 2007.

[15] R. Courant, Variational methods for the solution of problems of equilibrium and vibrations, Bull. Amer. Math. Soc., 49 (1943), 1-23.

[16] P. Destuynder, M. Jaoua, and H. Sellami, A dual algorithm for denoising and preserving edges in image processing, J. Inverse. Ill-Posed. Probl., 15(2) (2007), 149-165.

[17] W. M. Feng, P. Yu, S. Y. Hu, Z. K. Liu, Q. Du, and L. Q. Chen, Spectral implementation of an adaptive moving mesh method for phase-field equations, J. Comput. Phys., 220(1) (2006), 498-510.

[18] T. P. Gurholt and X. C. Tai, 3D multiphase piecewise constant level set method based on graph-cut minimization, Numer. Math. Theor. Meth. Appl., 2 (2009), 403-420.

[19] Y. Huang, M. K. Ng, and Y. W. Wen, A fast total variation minimization method for image restoration, Multi. Model. Sim., 7 (2008), 774-795.

[20] M. Kass, A. Witkin, and D. Terzopoulos, Snakes: active contour models, Int. J. Comput. Vis., 1(4) (1988), 321-331.

[21] C. Li, C. Xu, C. Gui, and M. Fox, Level set evolution without re-initialization: a new variational formulation, Proc. CVPR. IEEE., Citeseer, 2005.

[22] F. Li and M. K. Ng, Kernel density estimation based multiphase fuzzy region competition method for texture image segmentation, Commun. Comput. Phys., 8(3) (2010), 623-641.

[23] F. Li, M. K. Ng, T. Zeng, and C. Shen, A multiphase image segmentaion method based on fuzzy region competition, SIAM J. Imaging Sciences, 3(3) 2010, 277-299.

[24] H. W. Li and X. C. Tai, Piecewise constant level set method for multiphase motion, Int. J. 
Numer. Anal. Model., 4(2) (2007), 291-305.

[25] J. Lie, M. Lysaker, and X. C. Tai, A binary level set model and some applications to MumfordShah image segmentation, IEEE Trans. Image. Proc., 15(5) (2006), 1171-1181.

[26] J. Lie, M. Lysaker, and X. C. Tai, A variant of the level set method and applications to image segmentation, Math. Comput., 75(255) (2006), 1155-1174.

[27] T. Lu, P. Neittaanmaki, and X. C. Tai, A parallel splitting up method and its application to Navier-Stokes equations, Appl. Math. Lett., 4(2) (1991), 25-29.

[28] T. Lu, P. Neittaanmaki, and X. C. Tai, A parallel splitting up method for partial diferential equations and its application to Navier-Stokes equations, RAIRO Math. Model. Numer. Anal., 26(6) (1992), 673-708.

[29] Z. Luo, L. Y. Tong, J. Z Luo, P. Wei, and M. Y. Wang, Design of piezoelectric actuators using a multiphase level set method of piecewise constants, J. Comput. Phys., 228 (2009), 2643-2659.

[30] G. I. Marchuk, Splitting and alternating direction methods, Handb. Numer. Anal. I., pages 197-462, North-Holland, Amsterdam, 1990.

[31] D. Mumford and J. Shah, Optimal approximations by piecewise smooth functions and associated variational problems, Comm. Pure. Appl. Math., 42(5) (1989), 577-685.

[32] S. Osher and R. P. Fedkiw, Level Set Methods and Dynamic Implicit Surfaces, Springer Verlag, 2002.

[33] S. Osher and J. A. Sethian, Fronts propagating with curvature dependent speed: algorithms based on Hamilton-Jacobi formulations, J. Comput. Phys., 79(1) (1988), $12-49$.

[34] Z. J. Rong, L. L. Wang, and X. C. Tai, Adaptive wavelet collocation methods for image segmenation using TV-Allen-Cahn models, UCLA CAM-Report, 09-91, 2009.

[35] L. Rudin, S. Osher, and E. Fatemi, Nonlinear total variation based noise removal algorithms, Phys. D., 60(1-4) (1992), 259-268.

[36] J. Shen and X. F. Yang, An efficient moving mesh spectral method for the phase-field model of two-phase flows, J. Comput. Phys., 228(8) (2009), 2978-2992.

[37] G. Strang, On the construction and comparison of difference schemes, SIAM J. Numer. Anal., 5 (1968), 506-517.

[38] J. C. Strikwerda, Finite Difference Schemes and Partial Differential Equations, Society for Industrial Mathematics, 2004.

[39] X. C. Tai and T. F. Chan, A survey on multiple level set methods with applications for identifying piecewise constant functions, Int. J. Numer. Anal. Model., 1(1) (2004), 25-47.

[40] X. C. Tai, O. Christiansen, P. Lin, and I. Skjælaaen, Image segmentation using some piecewise constant level set methods with MBO type of projection, Int. J. Comput. Vis., 73(1) (2007), 61-76.

[41] X. C. Tai and C. Wu, Augmented Lagrangian method, dual methods and split Bregman iteration for ROF model, In Scale Space and Variational Methods in Computer Vision-LNCS 5567, pages 502-514, Springer, Berlin, 2009.

[42] L. N. Trefethen, Spectral Methods in MATLAB, Society for Industrial and Applied Mathematics (SIAM), Philadelphia, PA, 2000.

[43] Y. Wang, J. Yang, W. Yin, and Y. Zhang, A new alternating minimization algorithm for total variation image reconstruction, SIAM J. Imaging. Sci., 1(3) (2008), 248-272.

[44] J. Weickert, B. Romeny, and M. A. Viergever, Efficient and reliable schemes for nonlinear diffusion filtering, IEEE Trans. Image. Proc., 7(3) (1998), 398-410. 